\title{
La economía social y solidaria de la BioFeria de Miraflores
}

Recibido: 12/10/2016

Aprobado: 21/11/2016
Keyko Chuspe Apaza, Cristhian Ñañez Campos, Óscar Benites Bonifacio, Carmen Soria Núñez Universidad Nacional Mayor de San Marcos < keykochuspea@gmail.com >

\section{RESUMEN}

El presente documento es el resultado de un trabajo de investigación/acción grupal que busca examinar las dinámicas de producción y comercialización desenvueltas en la Bioferia de Miraflores, en la ciudad de Lima. Hace énfasis en cuatro aspectos: Su historia (memoria de la experiencia), la relación interna (tipo de organización), relación externa (con el entorno de la Bioferia) y la proyección que tiene la Bioferia a mediano y largo plazo. En el transcurso del examen se va a poder conocer y encontrar elementos propios de las economías sociales y solidarias, llamando la atención este tipo de desarrollo, que busca ubicarse fuera de la lógica neoliberal de mercado y se plantea como una alternativa a tomarse en cuenta dentro de la racionalidad capitalista en la que estamos inmersos incluso en lo que respecta a la academia. Este examen permite continuar discutiendo sobre las economías sociales y solidarias como una alternativa de producción a la impuesta desde el libre mercado. Así como conocer racionalidades distintas que parten de la producción económica como el tipo de organización que esta demanda, siendo en este caso la soberanía alimentaria y la comercialización ecológica en función de productos orgánicos, lo necesario a rescatar.

Palabras Clave: Soberanía alimentaria, economías solidarias, producción orgánica

\section{The social and solidary economy of the BioFeria Miraflores}

\begin{abstract}
This document is the result of a research / action group. The work which seeks to examine the dynamics of production and marketing in the Bioferia de Miraflores, in Lima city. It emphasizes four aspects: a. its history (organizational memory), b. internal relationship (type of organization), c. external relationship (with the environment Bioferia) and d. the projection having the Bioferia medium and long term. During the exam will be able to know and find elements of social and solidarity economies, drawing attention to this kind of development, which seeks located outside the neoliberal market logic and is presented as an alternative to be considered within of capitalist rationality in which we are immersed even in regard to the academy. This test allows to continue discussing the social and solidarity economy as an alternative to production imposed by the free market. As well as to meet different rationales that are based on economic output as the type of organization that this demand, which in this case food sovereignty and ecological marketing based on organic products, you need to rescue.
\end{abstract}

KEYwORDS: Food sovereignty, solidarity economies, organic production. 


\section{Introducción}

$\mathbf{E}$ 1 mundo globalizado está viviendo la configuración de una nueva geografía de centros y márgenes (Sassen, 2007: 143), proceso que consiste en la concentración, por un lado, de la vorágine de la economía financiera, el desarrollo de las tecnologías y los organismos multinacionales (como además todas las personas empleadas en estos sectores) así como la marginalización de un gran sector de la población que no ingresa o es expulsada de esta economía globalizada, entre ellos dos actores principales: inmigrantes y mujeres.

En nuestro país el Perú esto no nos es ajeno y particularmente en Lima esta dinámica de la globalización se vislumbra con los grandes centros financieros e hipermercados como Tottus, Metro, etc., basado en una comercialización de libre mercado, y que expende en su mayoría productos tratados químicamente y sin algún reparo de que sea beneficioso para la salud humana. Se conforman entonces grandes empresas oligopólicas del rubro de la comercialización de productos de "pan llevar" los cuales están ganando cada vez más terreno ya no solo concentrándose en el centro de la ciudad o distritos de mayores recursos, sino también en los llamadas "Limas". En este proceso se va reconfigurando los hábitos de consumo de las personas, las cuales proporcionalmente a la expansión de estos grandes mercados, elevan su nivel de consumo en estos mismos espacios, lo que sin duda afecta a los pequeños comercios o mercados cercanos a las grandes tiendas comerciales.

Ante ello es necesario discutir si este patrón de consumo que se impone cada vez más es inevitable y el modo hegemónico de producir y comercializar alimentos producto de esta globalización no puede ser reemplazado; es pertinente al haber llegado a este punto plantear el concepto de la economía social y solidaria.

La economía social y solidaria -siguiendo a Coraggio (2011) - ve la posibilidad de desarrollar una socioeconomía, donde los agentes económicos no son escindidos de sus identidades sociales, mucho menos de su historia y de su incrustación en el mundo simbólico e institucional que denominamos como cultura. Pone en este sentido límites sociales al mercado capitalista y, si es posible, busca construir mercados donde los precios y las relaciones resultan de una matriz social que pretende la integración de todos con un esfuerzo y unos resultados distribuidos de manera más igualitaria (2011: 44-45).
Es evidente que esta lectura de la economía abre una discusión y una serie de preguntas necesarias de responder. Para ello, realizamos el estudio de un caso específico: La experiencia de la BioFeria situada en el distrito de Miraflores, exactamente ubicada en el parque Reducto número 2, entre las calles 15 de enero y Ramón Ribeyro con la avenida Paseo de la República, teniendo como referencia a la avenida Benavides cruce con la Vía Expresa.

La BioFeria de Miraflores se encuentra dentro del rubro de comercialización de alimentos orgánicos, por ello, basándonos en Nova (s.f.), asumimos que el mercado de productos orgánicos constituye un importante espacio, por la potencialidad que encierra, dado los actuales bajos volúmenes de ventas con relación a las ventas totales de productos alimenticios y los establecimientos especiales donde se ofertan, los cuales por lo general no son de fácil acceso para todos los consumidores (s.f.: 8)

A su vez la Organización de las Naciones Unidas para la Agricultura y la Alimentación (s.f.). Anota que las actividades de agricultura urbana y peri urbana son actividades que incluyen la producción o transformación de productos agrícolas y agropecuarios para autoconsumo, así como su comercialización, aprovechando eficiente y sosteniblemente recursos e insumos locales, respetando los saberes y conocimientos locales y promoviendo la equidad de género a través del uso y coexistencia de tecnologías apropiadas y procesos participativos para la mejora de la calidad de vida de la población urbana y la gestión urbana social y ambiental sustentable de las ciudades (s.f.: 2).

El estudio de la BioFeria de Miraflores requiere que tengamos información que nos ayude a establecer qué relaciones desenvuelven los productores y cómo comercializan su producción. Entender qué es una feria de productos alimenticios naturales y el proceso por el cual esta se organiza, donde los productos no son presentados por su marca sino valorados por su proceso de producción y la calidad que adquieren para sus consumidores. Además de darnos una serie de pistas de por qué y cómo este tipo de ferias surgen y se ubican en ciertos espacios de una ciudad de las característica de Lima.

Para lograr estos objetivos propuestos, ha sido necesario visitar periódicamente la BioFeria de Miraflores para observar cuales son las dinámicas de comercialización observadas, así como conocer desde los mismos productores y los responsables de la BioFeria el testimonio que nos puedan brindar en función de conocer el nivel de conciencia que tienen sobre esta apuesta de 
producción y comercialización basado en lo orgánico. Fue una tarea al inicio complicada pero se logró obtener las fuentes necesarias para llevar a cabo esta indagación gracias al apoyo de Charito Pinto, coordinadora de la BioFeria, así como de los productores que accedieron a darnos información mediante las entrevistas realizadas.

\section{Historia y memoria de la BioFeria de Miraflores}

\subsection{Breve historia}

¿Cuál es la importancia de la Agricultura ecológica? Centro Ideas (2016) señala: "La mayoría de personas podría argumentar que esta se encuentra en la producción de alimentos saludables que no debilitan la salud humana ni dañan el ecosistema." (2016: 3). Claro que es importante señalar ese aspecto pero no es la única característica pues Nova (s.f.) señala: "La aplicación y desarrollo de una agricultura ecológica (...) que además de considerar el aspecto ecológico incluye los aspectos económicos sociales, en busca del mejoramiento del nivel de vida de los que la aplican, y el logro de la auto sustentabilidad del sistema productivo." (s.f.: 12). La segunda característica es la que consideramos esencial para este trabajo y puede verse reflejada en la historia de la pequeña feria que se desarrolla en el Parque el Reducto número 2 y que tiene por nombre "BioFeria de Miraflores"

Charito Pinto, coordinadora de la BioFeria de Miraflores, testimonia que esta iniciativa surge el 4 de diciembre de 1999 de la necesidad de los productores ecológicos de buscar espacios para poder ofertar sus productos al público. ${ }^{1}$ Así, la BioFeria pasó a ser el punto de promoción: del valor de los alimentos ecológicos, de la importancia de la biodiversidad y sobre todo de una vida integral y sana basada en la armonía a pesar de las diversas posturas (Wú Guin, 2014).

\subsection{Antecedentes}

En el ańo 1989 se realizó el primer Encuentro $\mathrm{Na}$ cional de Agricultura Ecológica (ENAE), este estaba presidido por tres organizaciones no gubernamentales: IDMA, Centro IDEAS y CARPER, el objetivo fue articular diversas manifestaciones en agricultura con criterios ecológicos en una propuesta integral, como,

1 Entrevista a Charito Pinto, coordinadora de la BioFeria de Miraflores, realizada el 11 de junio del 2016. conservación de suelos, cultivos andinos, uso de compost, agroforestería, biohuertos, agricultura ecológica. El centro de atención giró alrededor de las bases conceptuales y científicas de la agricultura ecológica, así como, la revisión de algunas experiencias agroecológicas en costa y sierra, además de la definición de un plan de acción conjunto. Al realizarse el tercer ENAE, en el año 1992, surge la necesidad de la creación de una certificadora de productos ecológicos cuyo fin seria impulsar la producción para el mercado. ${ }^{2}$

Este, sin duda, es un aspecto resaltante y nos permite concluir que el proceso de articulación por el cual pasaban los agricultores ecológicos tenía como fin la producción destinada al mercado, motivo por la cual necesitaban de un proceso de certificación que les diera garantía a sus productos y así estos pudieran ser distribuidos. Al respecto Nova (s.f.) señala: "Para que los productos resultantes de la agricultura orgánica sean reconocidos como tal por el mercado requieren de una certificación (deben pasar por un proceso de inspección, verificación y certificación), que avale que los productos calificados como orgánicos se producen efectivamente de conformidad con los principios de la agricultura orgánica.” (s.f.: 12)

El quinto y sexto ENAE realizados en abril de 1996 y julio de 1998, respectivamente, tuvieron como preocupación y objetivo principal la certificación, comercialización, producción y masificación de productos ecológicos a través de la implementación de ferias ecológicas locales. Fue también en el año 1998, como parte de una iniciativa tomada por el Centro IDEAS, que se crea el Grupo Ecológica Perú, como una institución que promueve los mercados ecológicos locales. $\mathrm{Al}$ año siguiente, en el mes de diciembre, empezó el funcionamiento de la primera BioFeria. ${ }^{3}$

\subsection{Desde sus inicios a la actualidad}

Con el lema "El gusto de vivir sano" la BioFeria de Miraflores abre sus puertas al público el 4 de diciembre de 1999, tras un esfuerzo de 11 productores, que se dividían en asociaciones de productores, productores

2 RAE Perú, 1989 - 2008: Del primer al decimoprimer ENAE. Página web Centro IDEAS.

http://www.ideas.org.pe/index.php/agricultura-ecologica/ item/230-rae-peru-1989-2008-del-primer-al-decimoprimer-enae

3 RAE Perú, 1989 - 2008: Del primer al decimoprimer ENAE. Página web Centro IDEAS.

http://www.ideas.org.pe/index.php/agricultura-ecologica/ item/230-rae-peru-1989-2008-del-primer-al-decimoprimer-enae 
independientes y algunos otros que pertenecían a diversas ONG, como en el caso de Silvia Wú y Fernando Alvarado pertenecientes al Centro Ideas, en colaboración con la gestión del entonces alcalde Fernando Andrade Carmona.

La relación de miembros de la BioFeria no ha sido constante. Charito Pinto señala que, desde su conformación hasta la actualidad, algunos productores y ONG's han ido dando un paso al costado; pero que muchos otros se han ido sumando. La diferencia es que estos ahora son enteramente independientes. Por ello, Ecológica Perú es concebida como una asociación de productores independientes.

El requisito principal que se les pidió a estos nuevos productores fue la certificación. Ella menciona que exigían a sus productores que estuviesen certificados pero la BioFeria no tiene relación con ningún tipo de certificadora. Pinto menciona: "Cada productor busca su certificadora, nosotros no podemos obligarlos a que lo hagan con una entidad en especial. ${ }^{4}$

La gestión que tienen del espacio está en función de la auto-organización de los productores, además que el espacio con el que cuentan es mínimo y no puede ser ampliado. Por eso, Pinto indica que no está entre los planes de la BioFeria admitir más productores. Ella lo dice en sus propias palabras: "No podemos admitir más productores dentro de la feria ya que como ven el espacio con el que contamos es muy reducido, nosotros cuidamos este espacio como nuestro, ya que si hay alguna rajadura en la vereda, nosotros mismos contratamos al personal para que la repare, la Municipalidad de Miraflores está totalmente desentendida al respecto."

La relación de la BioFeria con la Municipalidad de Miraflores se inició, como fue mencionado antes, en la gestión del alcalde Fernando Andrade Carmona en 1999. Él aceptó el proyecto y brindó el aval, junto al espacio requerido para su realización, además de otros recursos. Cuando terminó el periodo de mandato de Andrade, la BioFeria perdió contacto con la Municipalidad de Miraflores y paso por un periodo de informalidad y conflictos con esta institución.

Fue recién en el periodo de gestión del alcalde Manuel Masías Oyanguren, entre el 2007 y 2010, en donde se obtuvo la aceptación total y se les brindaron todo tipo de facilidades; pero nuevamente, una vez que este alcalde termina su periodo de mandato, la relación que se tenía con la municipalidad se volvió a resquebrajar.

Como seńala Pinto: "Actualmente nos encontramos aquí por una suerte de costumbre, no hemos gestionado ningún permiso, lo que hace la municipalidad es mandarnos algunos policías y serenazgos para que cuiden." 6

Desde enero del año 2003, por medio de la gestión de Ecológica Perú, la mayoría de los productores de la BioFeria de Miraflores distribuyen sus productos a través de Supermercados peruanos S.A7. Esto sin duda es una característica principal de las economías sociales y solidarias. Citando a Coraggio:

Esta economía social (...) debe competir por las voluntades que orientan las decisiones económicas individuales y también competir con las organizaciones capitalistas en sus mercados, sin caer para ello en la objetivación propia de la empresa capitalista, que ve a las personas como sustituibles y sus necesidades como un gancho para incentivarlas a contribuir a la eficiencia empresarial. (Coraggio, 2011: 44).

Como se mencionó en un inicio, actualmente sólo productores independientes forma $\mathrm{n}$ parte de la BioFeria de Miraflores. Pinto comenta además que recientemente han tenido problemas legales con el Centro IDEAS, debido a que el nombre de "BioFeria" esta patentado por Ecológica Perú; pero este había sido utilizado por el Centro ideas para organizar una feria en La Molina, con lo cual se deduce que el Centro Ideas ya no participa en la gestión de Ecológica Perú ni tampoco tiene injerencia en la BioFeria de Miraflores.

Actualmente la BioFeria cuenta con 44 stands y funciona todos los sábados desde las 9 a.m. hasta las 3 p.m. aproximadamente, "El gusto de vivir sano", en palabras de Charito Pinto, implica elegir lo que comes y el comer bien.

Antes de finalizar este apartado es necesario recalcar que si bien en sus inicios Ecológica Perú fue concebida como una suma de voluntades, gracias al aporte de organizaciones no gubernamentales y productores independientes, en la actualidad su naturaleza puede ser entendida como una asociación de productores, esta asociación no guarda relación con las ONG, son enteramente independientes, de aquí que podemos esbozar una de sus características más importantes: La autogestión, que se manifiesta en diversos aspectos, el 
funcionamiento de la BioFeria es uno de ellos, y tiene su núcleo en la articulación de los productores.

\section{Análisis interno}

\subsection{Organización y conflictos internos}

Si observamos la organización de la BioFeria de Miraflores notaremos que es una organización no muy compleja, con determinada cantidad de stands (44), con una marca registrada (Ecológica), y que produce $\mathrm{y}$ vende (también, para grandes tiendas y supermercados); podríamos inferir que sigue una lógica de mercado. Sin embargo, haciendo un estudio más profundo de este caso salen a relucir elementos que pondrían en tensión esta afirmación a priori.

Entre estos elementos podemos encontrar dos esenciales:

a) La dinámica interna. La organización es en gran medida horizontal, porque si bien es una feria donde los productores y las productoras tienen autonomía en su espacio designado para la venta de sus productos (por lo que algunas veces vemos que un stand tiene mayor cantidad de elementos ofertados e incluso mayor personal en su espacio) y por consiguiente nos llevaría a pensar que hay productores o productoras más importantes que los y las demás, al momento de tomar decisiones sobre la feria y su desarrollo orgánico todos y todas tienen el mismo poder de decisión como asociados y asociadas a Ecológica (asociación de productores). Esto se da en las asambleas que tienen periódicamente. Ahí se democratiza la posición de cada una y cada uno frente al resto. Ahora ya tocado el punto de las asambleas también es pertinente señalar que a través de este mecanismo de participación directa se elige a su junta directiva, la cual cumple funciones por dos años. En la actual junta, conformada por 6 miembros, es Cecilia Pardo del Pino la que ocupa el cargo de presidenta de Ecológica.

b) La diversidad de stands. Podemos encontrar en los stands desde emprendimientos ${ }^{8}$ hasta empresas medianas consolidadas ${ }^{9}$, los cuales se basan en los principios de lo ecológico ("la protección y respeto a la naturaleza por encima del negocio") -como

8 Como el stand de Surimanta, grupo de música autóctona aimara quienes venden CD's y accesorios afines a la cultura representada.

9 Como CRIECOIN dedicada a la venta de huevos orgánicos. comentaba Regulo Rosado, vendedor del stand de Surimanta-. También observamos, tomando el caso de CRIECOIN, que la conformación de las empresas es de corte mayoritariamente familiar, participando en la producción "hasta la nuera” -como señaló Jaime Suyo, propietario de esta empresa-, y donde está presente una racionalidad socieconómica que se fortalece en su estrecha vinculación con las unidades domésticas (Muñoz, 2010). Lo productivo y reproductivo no se ve por separado sino están íntimamente ligados. También en la comercialización (dentro de los stands) podemos observar la participación de varios miembros de la familia, como en Ekovida Organics, que se dedica a producir postres y dulces elaborados sin gluten ni lácteos, donde participan en la venta el padre como las hijas.

Organizaciones de este tipo, que privilegian formas de producción, comercialización y consumo que enfaticen en el trabajo y la reproducción, no están exentas de conflictos a nivel interno. Como advertimos, la BioFeria de Miraflores agrupa a un conjunto de productores de distintos rubros bajo la coordinación de la asociación de productores Ecológica Perú.

Esta última organización se encarga, básicamente, de labores de coordinación y asesoramiento a los distintos miembros que integran la BioFeria. Uno de los problemas principales se daba entre los vendedores y las vendedoras de los stands y Ecológica Perú -en los inicios, antes de ser una asociación plena-, el problema era de carácter económico, puesto que algunos miembros, al decir que Ecológica Perú era una ONG, enfatizaban en el hecho de que esta no tenía por qué cobrarles nada por los diversos servicios prestados, como administración de almacén, armado de los stands y diversos implementos necesarios para la instalación de la feria. Ahora tampoco, se encuentran de acuerdo con las tasas que se fijan, en su opinión, es demasiado para las tareas que se realizan con estos fondos. Ante estos problemas derivados del manejo del presupuesto, Ecológica ha optado por difundir el presupuesto durante las asambleas.

Otro problema se da en función de la incorporación de miembros nuevos, esta potestad, la de incorporar a nuevos miembros a la feria, también habilita a los miembros de Ecológica a regular la comercialización en los stands. Ecológica norma la oferta mediante el Reglamento de la BioFeria y una Ficha de Oferta 
que cada miembro debe llevar obligatoriamente en los stands. Esta ficha permite a los organizadores conocer los cultivos y variedades manejadas por cada productor. De esta manera, lo antes mencionado supone uno de los principales conflictos dentro de la organización, porque en muchas oportunidades los productores no cumplen con las distintas normas y lo más importante, no se limitan a vender los productos que la Ficha de Oferta los habilita.

Una de alternativas como solución a las posibles disputas entre los comerciantes y vendedores es el Reglamento de la BioFeria. Es aprobado en una Asamblea Extraordinaria y rige para el funcionamiento de la BioFeria organizada por Ecológica. Los miembros del Consejo Directivo son quienes se encargan de dar algunas modificaciones si es que la situación lo amerita. El último Reglamento fue aprobado el 19 de setiembre de 2015.

El Reglamento Interno ha sido fundamental a lo largo de toda la gestión y el funcionamiento de la BioFeria, sino los conflictos hubieran sido insalvables y actualmente las disputas serían mucho mayores. Tener un reglamento, una norma de funcionamiento hace que se pueda aplicar beneficios y sanciones de manera imparcial. Mientras se respete gran parte de los puntos, la continuidad de la BioFeria será armoniosa, aunque existan aún ciertas diferencias entre los distintos actores que forman parte de ella.

Volviendo a la idea inicial entonces es necesaria hacerse la pregunta ¿Persigue la BioFeria una lógica de mercado? ¿Su organización responde a esta?

Queda claro que una lógica totalmente de mercado no es, puesto que la apuesta en cuanto su producción y comercialización es una apuesta ecológica basándose en lo orgánico, que nos llevaría a pensar incluso -siguiendo a Jiménez (2015)- en la posibilidad que se dé una autonomía alimentaria "que se construye desde el momento en que cada persona toma consciencia del respeto que debe tener para con el medio ambiente y lo importante que es tener conocimiento de dónde viene el alimento que consume. Asumiendo el compromiso de un consumo responsable, un tejido comunitario se hace indispensable para instalar prácticas donde se cuide de sí mismo, del otro y de la tierra (agro-ecología)" (2015: 9). Los productores/vendedores siempre han mostrado en sus intervenciones la intención de ofrecer productos que vayan en armonía con la naturaleza y sean beneficiosos para la salud de las personas que lo consumen. Además el hecho que las mismas personas que producen sean las que vendan rompe con esta lógica de lucro propia de los intermediarios observados en las grandes tiendas comerciales y en general negocios que se mueven bajo la economía de libre mercado.

En cuanto a su organización, la participación democrática de sus miembros es totalmente necesario para la solidez de su asociación y por ello el mantenimiento de la BioFeria, y esto es posible porque "cuando un grupo se implica de manera directa en la solución de algo que se percibe como problemático, en este caso, la ausencia de una autonomía respecto a la producción y distribución de alimentos, se obtiene el fortalecimiento de los lazos sociales" (Jiménez, 2015: 5). Entonces ante una necesidad de comercializar productos orgánicos, y teniendo un mercado que los toma muy poco en cuenta, la cooperación y solidaridad entre estos productores emerge y se refuerza constantemente, lo que también se traslada al aspecto político, en cuanto su organización y nivel de participación como ya se señaló antes. Hasta este punto se concluye que la BioFeria de Miraflores si bien no está exenta de tener participación en el mercado de manera directa, por otra parte desarrolla redes de cooperación y solidaridad propias de una economía solidaria, dándonos como ejemplo que sí se puede apostar por otro tipo de producción y comercialización ética y armoniosa con la vida.

\section{Relaciones externas}

Para plantear una clasificación de los agentes que se desenvuelven en el entorno de la BioFeria de Miraflores tomamos en cuenta su ubicación espacial. A partir de aquí, identificamos a los agentes existentes en el lugar (Municipalidad de Miraflores, instituciones aledañas, vecinos del área) y analizamos las relaciones que establecen entre sí y con los productores de la BioFeria.

\subsection{El parque}

La BioFeria de Miraflores se ubica en un concurrido y conocido parque del distrito de Miraflores: El parque Reducto número 2. El cual representa un espacio emblemático para la historia local ya que en esta área se encontraba el segundo de los cinco reductos que defendieron a la ciudad de Lima durante la invasión chilena, producida durante la Guerra del Pacífico, y que participaron posteriormente en la Batalla de Miraflores. Un patrimonio que posee un valor como Santuario 


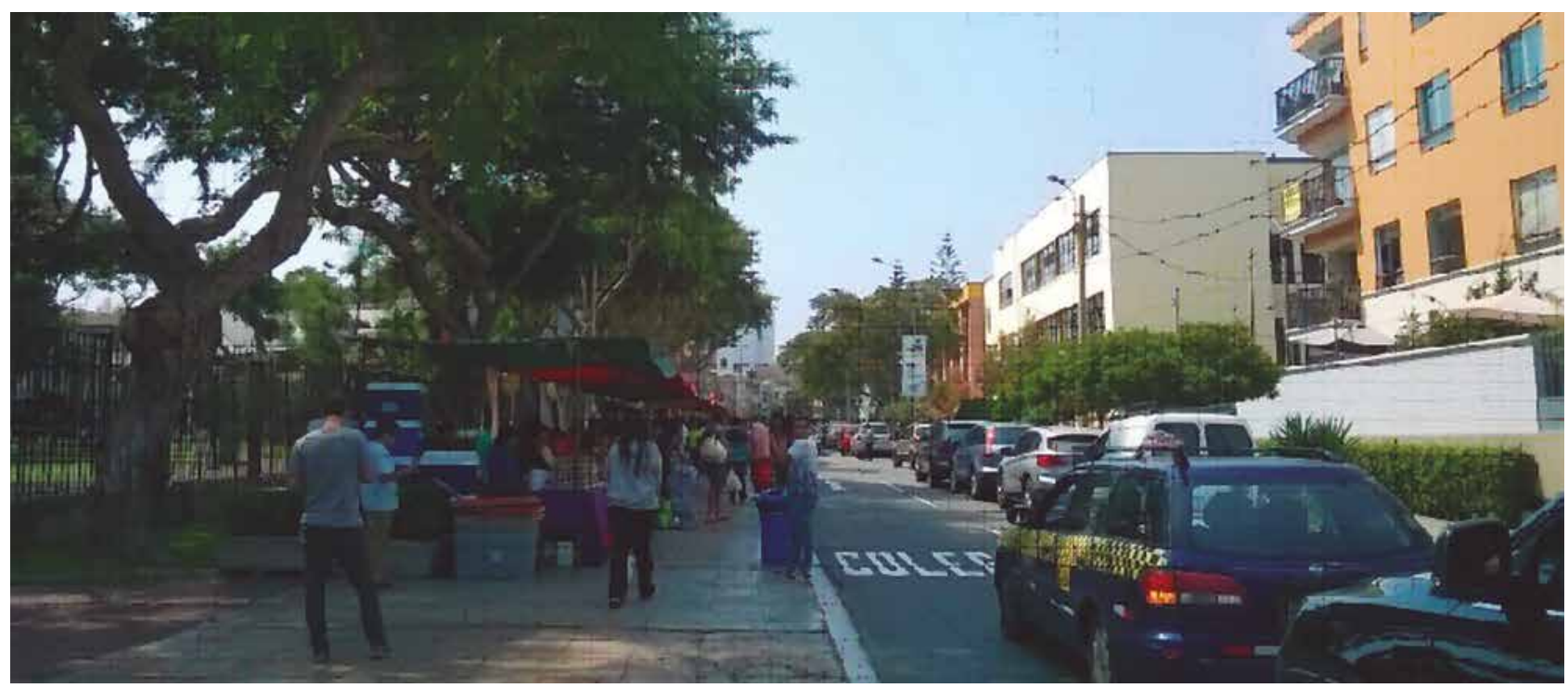

: Parque Reducto número 2, 2016 (Foto tomada por Keyko Chuspe)..

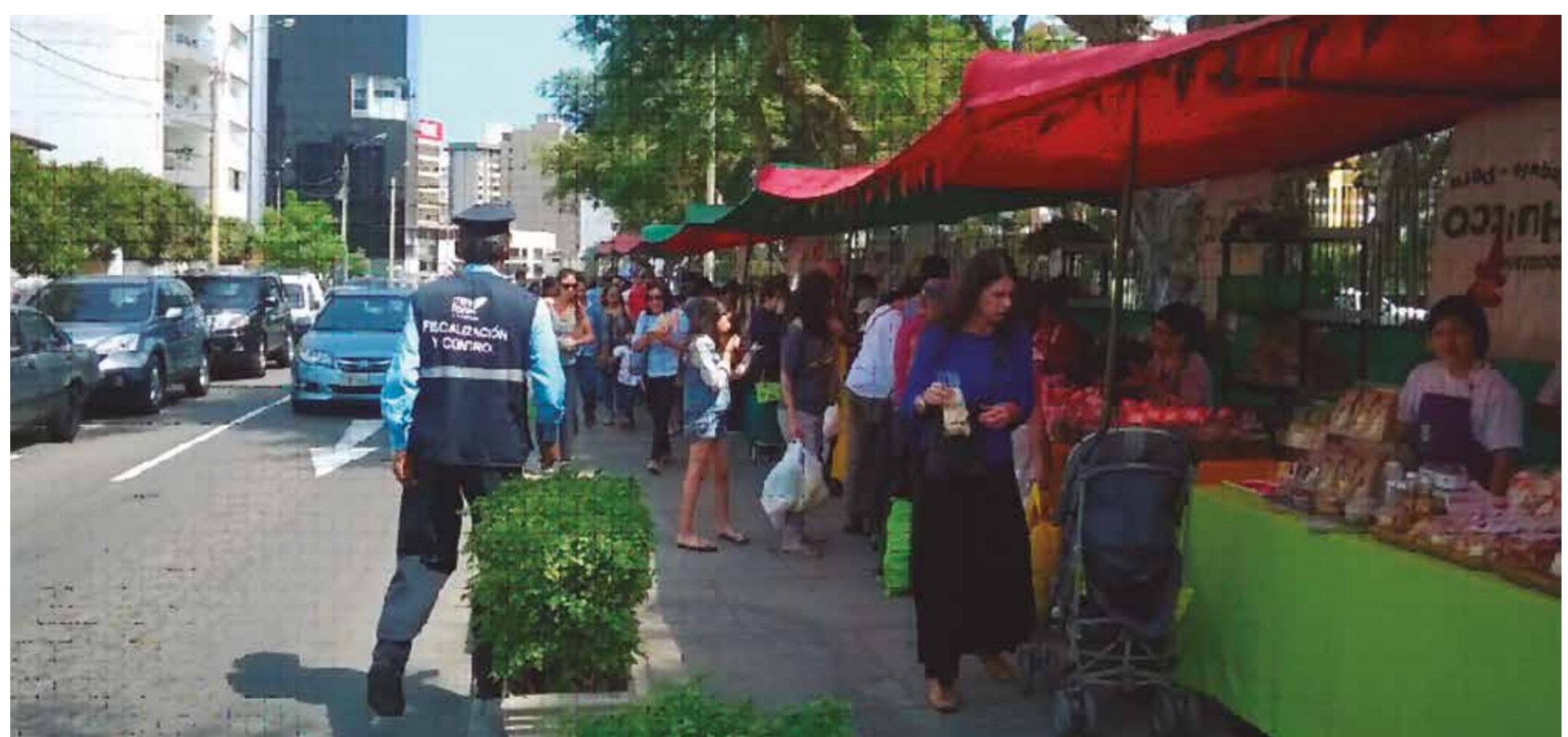

Imagen 2: BioFeria de Miraflores, 2016 (Foto tomada por Keyko Chuspe).

Histórico reconocido por la Municipalidad Distrital de Miraflores y declarado Monumento Nacional. Hoy en día podemos apreciar un museo de sitio así como una locomotora que antiguamente realizaba el recorrido férreo entre Lima y Chorrillos.

Este entorno logra integrarse perfectamente con la BioFeria, porque el tamaño de la infraestructura que representa, exige armar cada stand con cuidado para no perjudicar al parque o afectarlo, así como cuidar la limpieza del espacio que ocupa. Todos estos puntos la BioFeria los ha venido desarrollando de forma elabo- rada sin presentar un problema mayor, tanto para el parque como para el distrito en general. Jiménez señala al respecto:

De este modo, escapando a la lógica de producción actual, algunos colectivos se han encargado de producir los alimentos en espacios no convencionales, abriendo una importante alternativa para el autoconsumo y el comercio justo. (Jiménez, 2015: 3-4)

Como bien lo menciona Jiménez la BioFeria ha tomado esa idea, por ende se encuentra ubicada en el parque 
Reducto número 2 desde sus inicios hasta lo actualidad; pero esto también se debe a los permisos que la Municipalidad de Miraflores le otorgó, para brindarle las facilidades necesarias en su instalación cada sábado. Esto se puede apreciar en la presencia de serenazgos por parte de la municipalidad, ellos brindan seguridad tanto a los productores como a las personas que acuden a comprar, además de encargarse de dirigir el tránsito en las avenidas que rodean la BioFeria, porque en su mayoría las personas asisten en sus propios autos y por lo tanto se forma una especie de parqueo en los alrededores, que cuentan también con seguridad por parte de los serenazgos. Entonces, podemos decir que la relación que mantiene la asociación de productores Ecológica Perú con la municipalidad del distrito se da de la mejor manera, ya que son reconocidos; pero también la BioFeria, es decir, los productores en conjunto asumen la responsabilidad de encargarse de mantener limpio y ordenado toda el área que ocupan.

\subsection{Los clientes}

El público que acude a la BioFeria en su mayoría es perteneciente a "la clase media alta y clase alta", proveniente de distritos como Miraflores, el cual representa una cantidad elevada. Luego, en una menor cantidad, los asistentes vienen de distritos como Surco, Barranco y San Isidro. Un porcentaje también importante del público es extranjero que reside en la ciudad de Lima o está por razones de turismo, y visita el parque como un punto atractivo, debido a que cuenta con un museo de sitio y a su vez por ir de compras a la BioFeria.

La mayoría de ellos llega en transporte privado, como autos; un público joven lo hace en bicicletas, y es justamente este, que se encuentran en un rango de veinticinco a treinta y cinco ańos, el que más acude a la BioFeria. Además, es posible observar un importante porcentaje de mujeres, en comparación a los hombres, que concurren. Así como una considerable cantidad de visitantes adultos mayores, junto a niños y niñas, acompańados de mascotas caninas. A su vez es sencillo notar que la relación entre los vendedores y sus clientes se ha venido dando dentro de un tiempo previo, naciendo así los llamados "casero o casera", esto ejemplifica la trayectoria que ha tenido la BioFeria.

Las personas somos las que realmente habitamos las urbes: recorriendo las calles, las avenidas, los bulevares, etc.; ocupando los edificios, las plazas, las ram- blas, etc.; realizando infinidad de movimientos y de trayectos gracias a las piernas, las bicicletas, los autobuses y los metros. (Vivas i Elias, 2005: 18).

En este aspecto son los mismos clientes quienes se relacionan directamente con los productores de la BioFeria, ya que ambos son los principales actores que intervienen en la comercialización de los productos que se venden. Por lo tanto, son las personas en general quienes le dan el sentido de dinamismo a este tipo de espacios, donde prima el buen trato por parte de los productores, porque se puede distinguir su nivel de atención al cliente y calidez al estar en una constante adecuación a la exigencias del público que atienden. Así como el cuidado personalizado brindado al cliente extranjero y el uso del ingles durante las transacciones. Además de que los productores de la BioFeria están en una constante especialización y mejoramiento de los productos que ofrecen, por ende el respaldo que reciben de sus diversos clientes, sean desde los que ya forman parte de los "caseros" o de los nuevos que van uniéndose, engrosan las filas de los compradores y consumidores de sus productos y o en general del público que visita de manera creciente la BioFeria.

\subsection{El entorno}

El espacio que ocupa la BioFeria es un punto estratégico, puesto que presenta facilidades para llegar, uno de ellos es con el servicio del Metropolitano, específicamente en la estación Benavides. Otra alternativa es con transporte privado o público por la avenida Benavides entre las cuadras ocho y nueve. Esto posibilita que sea más accesible la llegada del público, además de contar con una dirección sencilla de ubicar y que representa un punto a favor para la BioFeria. Las facilidades para el ingreso, es decir, la zona que rodea a la BioFeria es muy concurrida de por sí. Su entorno incluye a la Iglesia Carmelitas y al colegio Nuestra Señora del Carmen, sin dejar de lado, la mención de las viviendas que colindan con el parque, las cuales tienen en su mayoría una cantidad de edificaciones de tipo departamental, con edificios que van de cuatro hasta los once pisos.

Lo más importante del espacio público, a juicio de las personas encuestadas, lo constituye también factores de tipos espacial, tales como la estética, el entorno, el equipamiento, las condiciones, etc. (Escobar, 2000:158). 


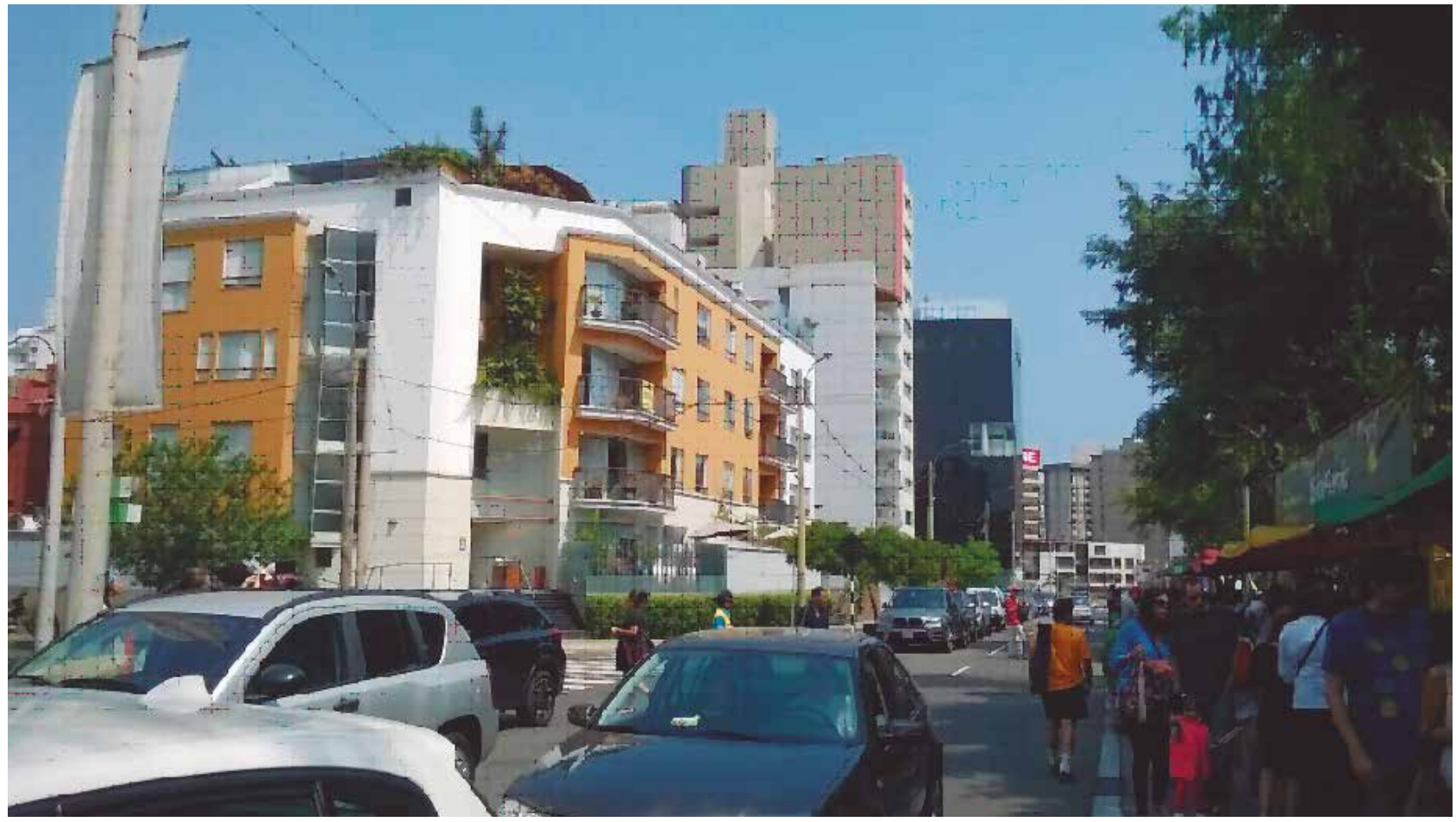

Imagen 3: Av. 15 de Enero, Miraflores, 2016 (Foto tomada por Keyko Chuspe).

Así como lo menciona Escobar, otro aspecto importante que tiene la ubicación de la BioFeria es la inLos objetivos de las y los productores, como integrantes de la BioFeria, muestran que desean un crecimiento, tanto para ampliar más ferias como para poder comercializar más productos, y que la idea de la producción y comercialización de productos orgánicos se extienda. Como se pueda observar en el siguiente comentario de una productora:

Nosotros como integrantes de la BioFeria tenemos como objetivo crecer, buscar más espacios donde podamos vender los productos y así crecer cada uno también, pero eso es un trabajo largo que toma también bastante tiempo ${ }^{10}$

Si bien están dispuestos a crecer, las y los productores consideran que este trabajo es progresivo y que se necesita apoyo para hacerlo.

En cuanto a la visión que tienen las y los productores, se proyectan en metas como organización y se puede observar que ellos tienen muy claro cómo funciona su comercio y que es lo que desean de aquí a algunos ańos. Como se puede evidenciar en la siguiente respuesta:

10 Entrevista realizada a Zhenia Ángela Blanco, productora de Valle Andino, integrante de la BioFeria de Miraflores, 2016.
Se visualiza como un negocio prominente y ejemplar en la producción, venta y promoción de productos de carácter ecológico; el fomento de la práctica de principios comerciales éticos, como el comercio justo $\mathrm{y}$ en el establecimiento de sistemas y modalidades de trabajo en sociedad con los integrantes de las cadenas productivas y comerciales que permitan el desarrollo de capacidades y el crecimiento económico e integral de todos, haciendo uso de prácticas de respeto mutuo y equidad. ${ }^{11}$

Ellas y ellos tienen claro que son una economía diferente y que respetan el ambiente, por lo tanto, se puede deducir que su propuesta esta ubicada como parte de una economía social y solidaria.

No obstante las y los productores muestran algunas dificultades que tienen aún para crecer, como la poca atención que se le da a la producción y comercialización de los productos orgánicos y que no existen muchos productores que promuevan este tipo de oferta productiva. Ellas y ellos buscan que esta idea llegue a todos y que se fortalezca más, pero aun con todas estas dificultades tienen una visión positiva sobre lo que esperan:

11 Entrevista realizada por un estudiante a Helena Manrique productora de Heliconias, integrante de la BioFeria de Miraflores. (2016) 
No obstante esta realidad, la tendencia es creciente hacia el fortalecimiento de las opciones que expresen un respecto por el planeta, sus recursos naturales y su medio ambiente, lo cual se convierte en una potencial fortaleza en el mediano y largo plazo. ${ }^{12}$

Además sumada a estas dificultades se observa que la relación con la Municipalidad de Miraflores no es tan buena. Como se mencionó al principio en cuento al papel de la municipalidad, en los inicio de la BioFeria de Miraflores se obtuvo apoyo de esta institución; pero en cuanto concluyó el periodo de los alcaldes, que apoyaron su iniciativa, esta relación no continuo más y así se ha venido dando con diferentes gestiones ediles. Lo que propone la seńora Pinto es volver a restablecer la comunicación con la municipalidad y afianzar los lazos que se habían construido, aunque teme que lo que se puede conseguir con el actual alcalde se rompa de nuevo acabada su gestión. Así no se espera mucho de la Municipalidad de Miraflores, sólo que se mantenga el apoyo en la seguridad como lo viene haciendo hasta ahora

La relación con el Estado en la promoción de productos ecológicos es deficiente y los productores sienten que es necesario ampliar esto y esperan que el Estado juegue un papel importante en la promoción y creación de políticas que les ayuden a fortalecer lo que hasta ahora han construido. Además ellas y ellos buscan con la promoción que el Estado haga tomar conciencia a la ciudadanía de la importancia que tienen, como factor fundamental para seguir difundiendo las ideas que propone la BioFeria, tal como lo propone Jiménez:

En este escenario, la búsqueda de alternativas de producción, distribución y consumo de alimentos resulta fundamental en aras de la sostenibilidad ecológica y orgánica. La idea de una nueva independencia implica la generación de un compromiso en el que las personas entiendan la urgencia de implicarse y responsabilizarse como ciudadanos activos en la tarea de respetar, preservar y cuidar la tierra y el alimento. (Jiménez, 2015: 3)

12 Entrevista realizada por un estudiante a Helena Manrique productora de Heliconias, integrante de la BioFeria de Miraflores. (2016)

\section{Bibliografía}

Nova, A. (s.f.). La producción y el mercado de los productos orgánicos en el mundo 2000-2005. Recuperado de: https://www.nodo50.org/cubasigloXXI/economia/ nova6_300903.pdf

Organización de las Naciones Unidas para la Agricultura y la Alimentación. (s.f.). Agricultura Urbana y periurbana en América Latina y El Caribe: una realidad. Recuperado de: http://www.fao.org/fileadmin/templates/FCIT/ PDF/Brochure_FAO_3.pdf

Manual básico de agricultura ecológica. Recuperado de: http://www.ciencias-marinas.uvigo.es/bibliografia_ambiental/agricultura_ecoloxica/Manual\%20Agricultura\%20Ecoloxica.pdf

Jiménez von Oertzen, N. (2015). "La nueva independencia: de la huerta urbana a la autonomía alimentaria”. Ponencia presentada a la conferencia internacional: Las luchas sociales por la tierra en América Latina. Un análisis histórico, comparativo y global, organizada el 24 y 25 de junio de 2015, por la Universidad Nacional Mayor de San Marcos, la Universidad de Gante y la Red Muqui.

Vivas i Elias, Pep. Mora, Martín. Vidal i Moranta. Rojas, Jesús. López, Oscar. Valera i Pertegàs. Pol i Urrutia. García, Noel. (2005). Ventanas en la ciudad: Observaciones sobre las urbes contemporáneas. Madrid: Editorial UOC.

Escobar Ángel, José Fernando (2000). De la ciudad y su espacio público. Manizales: Editorial Universidad de Colombia Sede Manizales.

Muñoz, R. (2011). "Análisis y desafíos de la economía popular urbana. Reflexiones a partir de análisis sectoriales y casos de estudios de la Región metropolitana de Buenos Aires", en: Urquieta, P. (Coordinadora). Ciudades en transformación. Disputas por el espacio, apropiación de la ciudad y prácticas de ciudadania, La Paz: CIDES Universidad Mayor de San Andrés.

Wú, G. (2014). Las bioferias y el desarrollo del mercado ecológico local en el Perú: cuatro experiencias de donde extraer inspiración. Centro Ideas. Recuperado de: http://www.ideas.org.pe/index. php/66-agricultura-ecologica-joomla-old/agriculturaecologica-joomla-old2/153-las-bioferias-y-el-desarrollodel-mercado-ecologico-local-en-el-peru-cuatro-experiencias-de-donde-extraer-inspiracion.

Coraggio, J. L. (2011). Economía social y solidaria. El trabajo antes que el capital. Quito: Fundación Rosa Luxemburgo.

SASSEN, Saskia (2012). "Ciudades globales: la recuperación del lugar y las prácticas sociales", en: Saskia Sassen Una sociología de la globalización, Buenos Aires: Katz Editores. 